\title{
Caractérisation et suivi isotopique des eaux de la nappe semi-captive du Maestrichtien de la Côte d'Ivoire
}

\author{
Yéï Marie Solange OGA ${ }^{1 *}$, Théophile LASM ${ }^{1}$, Marc MASSAULT ${ }^{2}$, Derving BAKA ${ }^{1}$, \\ Gabriel AKE ${ }^{1}$, Christelle MARLIN ${ }^{2}$, Jean BIEMI ${ }^{1}$ et Claude HILLAIRE-MARCEL ${ }^{3}$ \\ ${ }^{1}$ Laboratoire des Sciences et Techniques de l'Eau et de l'Environnement, 22 BP 582 Abidjan22 - Côte \\ d'Ivoire, Université d'Abidjan-Cocody, Côte d'Ivoire. \\ ${ }^{2}$ Laboratoire Interactions et Dynamiques des Environnements de surface, Université de Paris-Sud, Bât \\ 504, Orsay, France. \\ ${ }^{3}$ GEOTOP/Chaire UNESCO, UQAM, Montréal, Québec. \\ *Auteur correspondant, E-mail : oga_oms@yahoo.fr; Tel :00 $22507856246 / 02694931$; \\ Fax : 22522445270
}

\section{RESUME}

Les ressources en eau dans la région du Grand Abidjan sont tributaires des réserves souterraines, contenues essentiellement dans les formations sablo-argileuses du Continental Terminal (CT) et sableuses du Quaternaire. L'aquifère du Maestrichtien, situé à environ $200 \mathrm{~m}$ sous le CT, semble abriter de grandes réserves d'eaux souterraines qui peuvent être d'une importance stratégique; malheureusement les paramètres caractéristiques chimiques, isotopiques et hydrodynamiques (transmissivité, perméabilité, emmagasinement) de la nappe du Maestrichtien ne sont pas connus. Cette étude présente et discute les résultats des analyses chimiques (éléments majeurs) et isotopiques (oxygène-18, deutérium, tritium, carbone-14 et carbone-13) effectuées sur ces eaux à Abidjan, au Sud de la Côte d'Ivoire. Les eaux du Maestrichtien indiquent un pH presque neutre $(\mathrm{pH}=6,86)$, une forte alcalinité estimée à $205 \mathrm{mg} \cdot \mathrm{l}^{-1}$ et une minéralisation plus élevée (394 $\mu \mathrm{S} . \mathrm{cm}^{-1}$ ) par rapport à celles du CT et du Quaternaire. Les teneurs moyennes (1995/96) s'établissent pour l'oxygène-18 à $-3,6 \pm 0,1 \%$ ot pour le deutérium à $-17,7 \pm 1,6 \%$ avec un excès en deutérium de $10,6 \pm 0,2 \%$. L'activité ${ }^{14} \mathrm{C}$ du Maestrichtien est de $26,4 \pm 0,6 \mathrm{pCM}$ pour une teneur en ${ }^{13} \mathrm{C}$ de $-16,2 \%$ o vs V-PDB (août 1996). La présence d'eaux anciennes dans cet aquifère est confirmée par une teneur en ${ }^{3} \mathrm{H}$ inférieure au seuil de détection $(<0,8 \mathrm{UT})$. Les activités en ${ }^{14} \mathrm{C}(33,3 \pm 0,2 \mathrm{pCM})$ et les teneurs en ${ }^{13} \mathrm{C}(-14,73 \%$ ov VPDB $)$ sur les eaux prélévées en novembre 2008 semble indiquer un rajeunissement des eaux du Maestrichtien lié problablement aux pompages.

() 2011 International Formulae Group. All rights reserved.

Mots clés : Isotopes de l'environnement, Minéralisation, Recharge, Temps de séjour, Crétacé supérieur, Côte d'Ivoire.

\section{INTRODUCTION}

L'alimentation en eau potable des populations du Sud littoral ivoirien est tributaire des ressources en eaux souterraines captées principalement dans l'aquifère du Continental Terminal (CT). Cette partie de la Côte d'Ivoire connaît des problèmes d'approvisionnement en eau potable, de 
pollution nitratée des eaux souterraines en milieu urbain (Adjamé, Plateau, Capral et Jacqueville) et en milieu rural (Pass), (Kouadio et al., 1998; Oga, 1998 ; Jourda et al., 2006; Oga et al., 2007; Ahoussi et al., 2009) dus à l'intensification des prélèvements d'eaux et à l'urbanisation rapide et mal maîtrisée.

L'installation de nombreuses industries consommatrices d'eau, les usines textiles en particulier, l'amélioration du niveau de vie en général, et les perspectives de croissance démographique laissent prévoir des besoins de plus en plus importants.

L'aquifère du Maestrichtien, situé à environ $200 \mathrm{~m}$ en-dessous du CT, semble abriter de grandes réserves d'eaux souterraines qui peuvent être d'une importance stratégique pour fournir des sources d'approvisionnements alternatives afin de protéger la nappe du CT à Abidjan menacée actuellement par la pollution et la surexploitation.

Les paramètres hydrodynamiques de la nappe du Maestrichtien (transmissivité, perméabilité, emmagasinement) et les caractéristiques isotopiques des eaux ne sont pas connus en Côte d'Ivoire comparativement à l'aquifère du $\mathrm{CT}$ pour lequel les paramètres hydrauliques ( $\mathrm{T}, \mathrm{S}, \mathrm{K}, \mathrm{Q}, \mathrm{i})$, et les caractéristiques chimiques et isotopiques des eaux sont connus à travers plusieurs travaux (Oga, 1998; Oga et al., 2008; Adiaffi et al., 2009).

Le Maestrichtien (Crétacé Supérieur) est connu ailleurs en Afrique de l'Ouest, dans les bassins sédimentaires côtiers s'étendant du Sénégal au Nigéria. Ces formations du Crétacé sont caractérisées par un faciès détritique (sable, argile,) et par un faciès carbonaté composé de calcaires dolomitiques, de calcaires marneux, de marnes calcaires, de calcaires gréseux (Slansky, 1962; Bouzid, 1967; Loroux, 1978 ; Sow, 1992 ; Mott Mc Donald et al., 1993 ; Saos et al. (1993) in Coly 1996 ; Jorgensen et Banoeng-Yakubo, 2001, Akouvi et al., 2008 ; DGEA, 2009). Les paramètres hydrodynamiques du
Maestrichtien de l'Afrique de l'Ouest sont répertoriés dans le Tableau 1.

S'appuyant principalement sur les techniques de l'hydrochimie (ions majeurs) et de la géochimie isotopique $\left({ }^{18} \mathrm{O},{ }^{2} \mathrm{H},{ }^{3} \mathrm{H},{ }^{13} \mathrm{C}\right.$ et ${ }^{14} \mathrm{C}$ ), le Laboratoire des Sciences et Techniques de l'Eau et de l'Environnement (LSTEE) de l'Université de Cocody en collaboration avec le Laboratoire Interactions et Dynamique Environnements de Surface (IDES, Université de Paris-Sud) ont entrepris cette étude afin de comprendre le fonctionnement de cet aquifère en Côte d'Ivoire. Le but visé par l'étude est de décrire le faciès chimique des eaux souterraines du Maestrichtien, de préciser leur processus de minéralisation, d'indiquer les origines et mécanismes de recharge des eaux de la nappe et enfin, d'évaluer l'âge de ces eaux en Côte d'Ivoire.

\section{MATERIEL ET METHODES}

L'échantillonnage des eaux souterraines du Maestrichtien a porté sur l'ouvrage privé Awa de la SADEM de septembre 1995 à août 1996 et en novembre 2008. Les paramètres physiques (température, $\mathrm{pH}$, conductivité électrique et alcalinité) des eaux souterraines du Maestrichtien ont été mesurés in situ. L'eau pour les mesures in situ et pour les analyses a été prélévée directement sur le forage. Les analyses chimiques des éléments majeurs $\left(\mathrm{Na}^{+}, \mathrm{K}^{+}, \mathrm{Ca}^{2+}, \mathrm{Mg}^{2+}, \mathrm{Cl}^{-}, \mathrm{NO}_{3}^{-}\right.$, $\left.\mathrm{SO}_{4}{ }^{2-}\right)$, de la silice $\left(\mathrm{SiO}_{2}\right)$ et de l'aluminium ont été réalisées au Laboratoire IDE (Université de Paris-Sud), au laboratoire LANEMA et au Centre Ivoirien AntiPollution (CIAPOL) à Abidjan. L'incertitude sur les mesures de température, de $\mathrm{pH}$ et de conductivité électrique sont respectivement de $0,1^{\circ} \mathrm{C}$, de 0,02 unité $\mathrm{pH}$ et de $2 \mu \mathrm{S} . \mathrm{cm}^{-1}$.

Le diagramme de Piper a été utilisé pour la définition du faciès chimique des eaux. Par ailleurs, afin de préciser le processus primaire de minéralisation, le programme de modélisation thermodynamique PC Wateq Datagen 2.12 (Rollins, 1987) a été utilisé. Ce logiciel permet de calculer les indices de saturation et de déséquilibre, ainsi que la 
pression partielle des phases gazeuses $\left(\mathrm{CO}_{2}\right)$. L'évolution chimique de l'eau du Maestrichtien en fonction de son état d'équilibre ou de déséquilibre vis-à-vis des minéraux primaires et néoformés de la roche encaissante s'est faite suivant les équations d'équilibre de Fritz (1976), de Stumm et Morgan (1981). Les diagrammes d'équilibres partiels $\mathrm{Na}_{2} \mathrm{O}-\mathrm{Al}_{2} \mathrm{O}_{3}-\mathrm{SiO}_{2}-\mathrm{H}_{2} \mathrm{O}, \quad \mathrm{K}_{2} \mathrm{O}-\mathrm{Al}_{2} \mathrm{O}_{3}-$ $\mathrm{SiO}_{2}-\mathrm{H}_{2} \mathrm{O}$ et $\mathrm{CaO}-\mathrm{Al}_{2} \mathrm{O}_{3}-\mathrm{SiO}_{2}-\mathrm{H}_{2} \mathrm{O}$ ont été utilisés pour l'étude de l'interaction eauroche.

Les teneurs isotopiques des eaux ont été déterminées par différentes méthodes telles que la spectrométrie de masse $\left({ }^{18} \mathrm{O},{ }^{2} \mathrm{H},{ }^{13} \mathrm{C}\right)$, la spectrométrie de masse par accélérateur $\left({ }^{14} \mathrm{C}\right.$ AMS) et le comptage par enrichissement électrolytique $\left({ }^{3} \mathrm{H}\right)$ au Laboratoire IDES à Orsay. Le principe de mesure de l'oxygène-18 est basé sur la méthode classique d'équilibration isotopique (Epstein et Mayeda, 1953). Les teneurs en deutérium sont dosées selon Coleman et al. (1982). L'utilisation du carbone-14 en tant que chronomètre et traceur nécessite le dosage de l'isotope stable du carbone $\left({ }^{13} \mathrm{C}\right)$. Les incertitudes analytiques sur les mesures sont de $0,2 \%$ pour l'oxygène- 18 et le carbone- 13 et de $2 \%$ pour le deutérium.

\section{Presentation du site d'etude}

Le forage Awa de la Société Africaine d'Exploitation d'Eau Minérale (SADEM), sujet de cette étude est localisée dans la commune de Yopougon, une des dix communes du District d'Abidjan localisé entre les latitudes $5^{\circ} 10$ et $5^{\circ} 38$ Nord et les longitudes $3^{\circ} 45$ et $4^{\circ} 21$ Ouest (Figure 1).

Avec un taux de croissance de 3,7\% par an et une densité de population de 1475 $\mathrm{hab} / \mathrm{km}^{2}$, ce District regroupe une population estimée en 1998 à environ 3125890 habitants. Le climat est de type équatorial de transition appellé aussi climat Attiéen avec quatre saisons. Ce régime climatique est marqué par une monotonie des conditions météorologiques : les pressions sont basses, à peu près constantes, les vents sont rares et faibles, les régimes thermiques sont peu contrastés avec des contrastes saisonniers faibles, les précipitations sont toujours abondantes, tombant sous forme d'averses, souvent violentes.

Du point de vue géologique, la zone d'étude appartient au bassin sédimentaire côtier, composé de formations post éburnéennes, inscrites dans un rectangle côtier entre les latitudes $5^{\circ} 00$ et $5^{\circ} 30 \mathrm{~N}$ et entre les longitudes $3^{\circ} 00$ et $6^{\circ} 00 \mathrm{~W}$ (Loroux, 1978). Sur le plan tectonique, le bassin sedimentaire est séparé en deux zones distinctes par la faille des lagunes:

- Une zone au Nord de l'accident où la couverture sédimentaire atteint rarement 300 $m$ d'épaisseur ;

- Et une zone au Sud où le bassin est contrairement profond; le socle s'enfonçant au-delà de 4000 à $5000 \mathrm{~m}$ sous les sédiments (Martin, 1973).

Trois types d'aquifères se distinguent dans la région d'étude selon les travaux de Guerin-Villeaubreuil (1962) et Leneuf et Berthelot (1966) :

- L'aquifère du Quaternaire constitué par les sables marins grossiers (nappe du Nouakchottien) et les sables fins à grossiers (nappe de l'Oogolien) ;

- L'aquifère du Continental Terminal (CT) composé des sables graveleux (nappe du CT1), des sables grossiers fluviatiles (nappe du CT3) et des sables argileux (nappe du CT4). L'aquifère du CT repose en discordance sur l'aquifère du Maestrichtien (figure 2). Les travaux antérieurs de Loroux (1978) indiquent que le CT alimente la nappe du Crétacé supérieur par sa base. A l'état actuel des études, ce fait n'a pu être confirmé.

- L'aquifère du Maestrichtien où se loge la nappe du Crétacé Supérieur, nappe semi artésienne composée des bancs de calcaires gréseux, peu fissurés, de gros grains de quartz roulés et surmontant des niveaux coquilliers, d'âge crétacé supérieur (Loroux, 1978). La perméabilité, l'emmagasinnement, la transmissivité ainsi que le débit d'exploitation 
ne sont pas connus. Le niveau statique est à +2 m par rapport au niveau de la mer (Loroux, 1978; SOGREAH, 1996). La charge de la nappe du Maestrichtien par rapport au sol, ainsi que la différence de charge éventuelle avec le CT sont variables suivant que l'on se situe sur les hauts plateaux d'altitude 40 à 50 m ou 100 à $120 \mathrm{~m}$, représentés par les buttes du Continental Terminal au Nord de la lagune Ebrié. Des travaux sur les paramètres hydrodynamiques de l'aquifère du Maestrichtien sont en cours à l'Université d'Abobo Adjamé dans le cadre d'une thèse de Doctorat. Le forage Awa de la SADEM à Locodjro-Abidjan (Figure 3), d'une profondeur totale de $191 \mathrm{~m}$ et crépiné à partir de 156,7 $\mathrm{m}$ atteint cette formation en Côte d'Ivoire. L'eau captée est commercialisée en Côte d'Ivoire sous l'appellation "eau minérale AWA". Jusqu'à une date récente (2008), aucun suivi piézométrique de la nappe du Maestrichtien n'était effectué. Avec l'avènement du second forage de la Sadem captant le Maestrichtien, un enregistrement automatique du niveau de la nappe est effectué.

En Côte d'Ivoire, le Maestrichtien affleure à Eboinda au Sud-Est et sub-affleure à Yocoboué au Sud-Ouest (Aghui et Biémi, 1984).

\section{RESULTATS}

\section{Hydrochimie}

Les eaux du Maestrichtien indiquent une conductivité électrique de $394 \mu \mathrm{S} . \mathrm{cm}^{-1}$ et une alcalinité estimée à $205 \mathrm{mg} \cdot \mathrm{l}^{-1}$. Ces eaux ont un $\mathrm{pH}$ neutre $(\mathrm{pH}=6,86)$, en accord avec la nature carbonatée de l'aquifère, et sont relativement chaudes à la sortie du forage $\left(28,6^{\circ} \mathrm{C}\right)$. Le potentiel d'oxydo-réduction des eaux du Maestrichtien (+167 mV) témoigne des conditions faiblement oxydantes du milieu. La balance ionique de 6,0\% obtenue sur le prélèvement d'août 1996 est acceptable pour des eaux moyennement minéralisées.
Les eaux du Maestrichtien sont caractérisées par une minéralisation totale élevée de $331 \mathrm{mg} \cdot 1^{-1}$ ). Les ions dominants dans ces eaux sont les bicarbonates (89\%) pour les espèces anioniques et le calcium (77\%) pour les cations ; ce qui confère à ces eaux un faciès bicarbonaté calcique. Les teneurs en sodium représentent $19 \%$ de la minéralisation en cations. Les concentrations sont de 17,9 mg. $\mathrm{L}^{-1}$ en silice et de $0,1 \mathrm{mg} . \mathrm{L}^{-1}$ pour l'aluminium. Les eaux du forage Awa de la SADEM sont sous-saturées vis-à-vis de la dolomite $(-2,47)$ et proche de la saturation visà-vis de la calcite $(-0,35)$.

Sur les diagrammes de déséquilibre pour les 3 systèmes $\left(\mathrm{Na}_{2} \mathrm{O}-\mathrm{Al}_{2} \mathrm{O}_{3}-\mathrm{SiO}_{2}-\mathrm{H}_{2} \mathrm{O}, \mathrm{K}_{2} \mathrm{O}\right.$ $\left.\mathrm{Al}_{2} \mathrm{O}_{3}-\mathrm{SiO}_{2}-\mathrm{H}_{2} \mathrm{O}, \mathrm{CaO}-\mathrm{Al}_{2} \mathrm{O}_{3}-\mathrm{SiO}_{2}-\mathrm{H}_{2} \mathrm{O}\right)$ les eaux du Maestrichtien se situent dans le domaine de stabilité de la kaolinite.

\section{Géochimie isotopique}

Les résultats relatifs à l'isotopie sont consignés dans le Tableau 2. Les teneurs en isotopes stables $\left({ }^{18} \mathrm{O}\right.$ et $\left.{ }^{2} \mathrm{H}\right)$ des eaux du Maestrichtien varient de $-3,86$ à $-3,42 \%$ vs $\mathrm{V}$ SMOW en oxygène-18 et de $-20,03$ à $15,64 \%$ vs V-SMOW en deutérium. Les teneurs moyennes s'établissent pour l'oxygène-18 à $-3,6 \pm 0,1 \%$ ov $\mathrm{V}$-SMOW et pour le deutérium à $-17,7 \pm 1,6 \%$ vs $\mathrm{V}$ SMOW.

L'échantillon du Maestrichtien (août 1996) présente une teneur en ${ }^{13} \mathrm{C}$ de $-16,2 \%$ o $v s$ PDB, une activité de 26,4 \pm 0,6 pCM et une teneur en ${ }^{3} \mathrm{H}$ sous le seuil de détection $(<0,8$ UT). Les résultats obtenus par les différents modèles de correction sont indiqués dans le Tableau 2.

Les eaux prélevées en novembre 2008 indiquent des teneurs en ${ }^{18} \mathrm{O}=-3,61 \%$ o vs $\mathrm{V}$ SMOW et en ${ }^{2} \mathrm{H}=-19,36 \%$ os $\mathrm{V}-\mathrm{SMOW}$.

Les eaux de la SADEM prélévées en novembre 2008 indiquent une teneur en ${ }^{13} \mathrm{C}$ de $-14,73 \%$ ovs PDB et une activité ${ }^{14} \mathrm{C}$ de 33,3 +/- 0,2 pCM. 
Y.M. S. OGA et al. / Int. J. Biol. Chem. Sci. 5(3): 991-1004, 2011

Tableau 1 : Paramètres hydrodynamiques du Maestrichtien en Afrique de l'Ouest.

\begin{tabular}{|c|c|c|c|c|}
\hline Localisation & Piézometrie /Epaisseur (m) & $\begin{array}{l}\text { Transmissivité }\left(\mathrm{m}^{2} / \mathbf{s}\right) \\
\text { Emmagasinnement }(\%)\end{array}$ & Débit $\mathrm{m}^{3} / \mathrm{h}$ & Références \\
\hline $\begin{array}{l}\text { Togo } \\
\text { Secteur sud *entre la frontière du } \\
\text { Ghana et le Mono } \\
\text { *vers la frontière du Bénin, à l'est } \\
\text { de Tabligbo }\end{array}$ & $\begin{array}{l}\text {-Semi-captif captif non artésien } \\
-5 \text { à } 15 \mathrm{~m}\end{array}$ & $\begin{array}{l}1 \text { à } 610^{-3} \\
\text { Inconnu }\end{array}$ & $\begin{array}{l}\text { peut atteindre } \\
100 \mathrm{~m}^{3} / \mathrm{h}\end{array}$ & $\begin{array}{l}\text { Mott Mc Donald et al., } 1993 \\
\text { Akouvi, et al., } 2008\end{array}$ \\
\hline $\begin{array}{l}\text { Bénin } \\
\text { Affleure sur la bordure nord du } \\
\text { bassin }\end{array}$ & $\begin{array}{l}\text {-libre au Nord } \\
\text { en charge au Sud, artésien } \\
\text { jaillissant ou non } \\
-50 \mathrm{~m} \text { au Nord et }+800 \mathrm{~m} \text { au Sud } \\
\end{array}$ & $6,410^{-4}$ à $4,510^{-2}$ & $\begin{array}{l}50 \mathrm{~m}^{3} / \mathrm{h} \text { pouvant } \\
\text { atteindre } 100 \text { à } 150 \\
\mathrm{~m}^{3} / \mathrm{h}\end{array}$ & Slansky, 1962 ; Bouzid, 1967 \\
\hline Côte d'Ivoire & $\begin{array}{l}\text { Semi_captif artésien }(+2 \mathrm{~m}) \\
-50 \mathrm{~m}\end{array}$ & $\begin{array}{l}\text { Inconnu } \\
\text { Inconnu }\end{array}$ & inconnu & $\begin{array}{l}\text { Loroux, } 1978 \text {; Aghui et Biémi 1984; Sogreah } \\
1996\end{array}$ \\
\hline
\end{tabular}

La présence du Maestrichtien est confirmée dans le bassin de Keta au sud-est du Ghana, à la frontière avec le Togo (Jorgensen et Banoeng-Yakubo, 2001). Par contre, la recherche bibliographique entreprise n'a pas permis de confirmer la présence de cette nappe au Libéria.

Tableau 2: Résultats des analyses chimiques et isotopiques des eaux du Maestrichtien d'Abidjan (août 1996).

a) Paramètres chimiques, indices de saturation et de déséquilibre des eaux du Maestrichtien (août 1996).

\begin{tabular}{|c|c|c|c|c|c|c|c|c|}
\hline $\mathrm{Cl}^{-}$ & $\mathrm{NO}_{3}^{-}$ & $\mathrm{SO}_{4}^{-2}$ & $\mathrm{HCO}_{3}^{-}$ & $\mathbf{N a}^{+}$ & $\mathbf{K}^{+}$ & $\mathrm{Mg}^{2+}$ & $\mathrm{Ca}^{2+}$ & $\mathrm{Al}^{3+}$ \\
\hline \multicolumn{9}{|c|}{$\left(\mathrm{mg} \mathrm{l}^{-1}\right)$} \\
\hline 8,89 & 0,00 & 7,44 & 204,96 & 20,15 & 4,46 & 0,45 & 66,51 & 17,90 \\
\hline \multirow[t]{2}{*}{$\log \mathrm{PCO} 2$} & $\mathrm{SiO2}$ & MT & Anhydrite & Aragonite & Calcite & Dolomite & Gypse & Halite \\
\hline & $\left(\mathrm{mg.l}^{-\mathbf{1}}\right)$ & & $\mathrm{CaSO}_{4}$ & $\mathrm{CaCO}_{3}$ & $\mathrm{CaCO}_{3}$ & $\mathrm{CaCO}_{3}$ & $\mathrm{CaSO}_{4} 2 \mathrm{H}_{2} \mathrm{O}$ & $\mathrm{NaCl}$ \\
\hline$-1,86$ & 0,06 & 333,82 & $-2,83$ & $-0,49$ & $-0,35$ & $-2,47$ & $-2,65$ & $-8,34$ \\
\hline $\mathrm{SiO}_{2}$ amor & ISquartz & IAb-kao & IMusc-kao & IAn-kao & IMicr-kao & IKao-Gib & ICaM-kao & Iill-kao \\
\hline$-0,55$ & 0,78 & $-3,44$ & $-2,05$ & $-6,02$ & $-1,70$ & 1,17 & $-0,19$ & $-1,87$ \\
\hline
\end{tabular}

MT : minéralisation totale $; \mathbf{I S}$ : indice de saturation, concerne les minéraux carbonatés (calcite, dolomite, aragonite), sulfatés (gypse, anhydrite), les halogenures ( halite), le quartz et la silice amorphe ( $\mathrm{SiO}{ }_{2}$ amor) $; \mathbf{I}$ : indice de déséquilibre, concerne les minéraux primaires (albite, muscovite, microcline et anorthite) et les minéraux secondaires (gibbsite, kaolinite, montmorillonite calcique et illite) 
b) Teneurs en ${ }^{18} \mathrm{O}$ et ${ }^{2} \mathrm{H}$ des eaux du Maestrichtien à Abidjan.

\begin{tabular}{|c|c|c|}
\hline Date & $\delta^{18} \mathrm{O} \%$ vs V-SMOW & $\delta^{2} H$ \%ovs V-SMOW \\
\hline sept-95 & $-3,66$ & $-17,90$ \\
\hline oct-95 & $-3,68$ & $-15,64$ \\
\hline nov-95 & $-3,61$ & $-18,86$ \\
\hline déc-95 & $-3,86$ & $-17,44$ \\
\hline janv-96 & $-3,58$ & $-17,92$ \\
\hline févr-96 & $-3,47$ & $-17,98$ \\
\hline mars-96 & $-3,47$ & $-18,23$ \\
\hline avr-96 & non prélevé & non prélevé \\
\hline mai-96 & $-3,58$ & $-20,03$ \\
\hline juin-96 & $-3,45$ & $-16,93$ \\
\hline juil-96 & $-3,42$ & $-19,73$ \\
\hline août-96 & $-3,50$ & $-14,48$ \\
\hline nov -08 & $-3,61$ & $-19,36$ \\
\hline Moy 1995/96 & $-3,57$ & $-17,74$ \\
\hline E-Type 1995/96 & 0,13 & 1,63 \\
\hline
\end{tabular}

Les incertitudes analytiques sur les mesures sont de $0,2 \%$ pour l'oxygène- 18 et de $2 \%$ pour le deutérium. 
Tableau 3 : Temps de résidence corrigés des eaux du Maestrichtien.

\begin{tabular}{ll}
\hline Eichinger (1983) & $\mathbf{6 7 0 0}$ ans \\
\hline Evans et al. (1979) & 6880 ans \\
Tamers (1967) & 6980 ans \\
Ingerson et Pearson (1964) & 7100 ans \\
Fontes et Garnier (1979) & 7150 ans \\
Mook (1976) & 7260 ans \\
Fontes et Garnier équilibrant $_{\text {AIEA (Salem et al., 1980) }}$ & 8900 ans \\
\hline
\end{tabular}

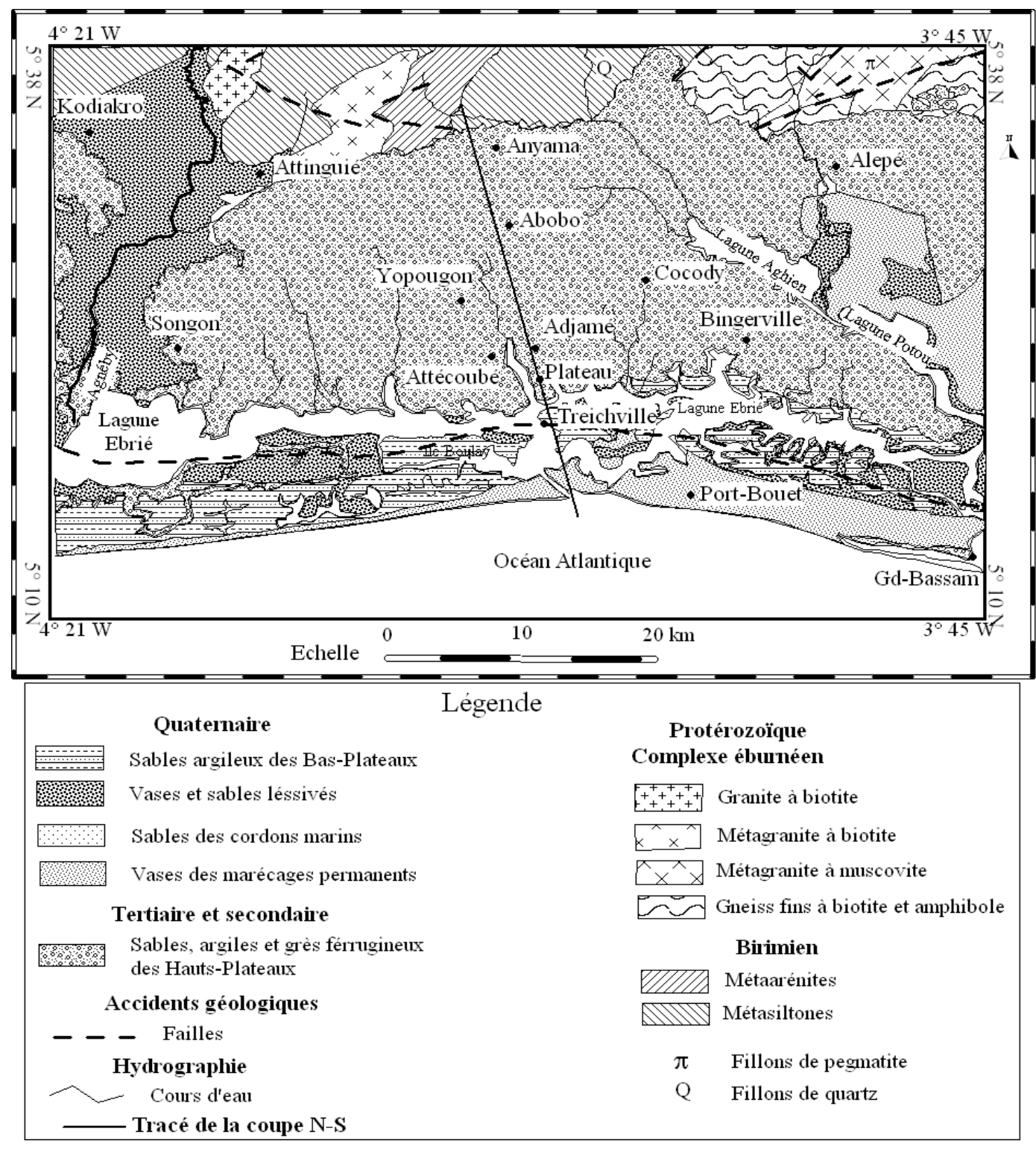

Figure 1 : Carte géologique du District d'Abidjan. 


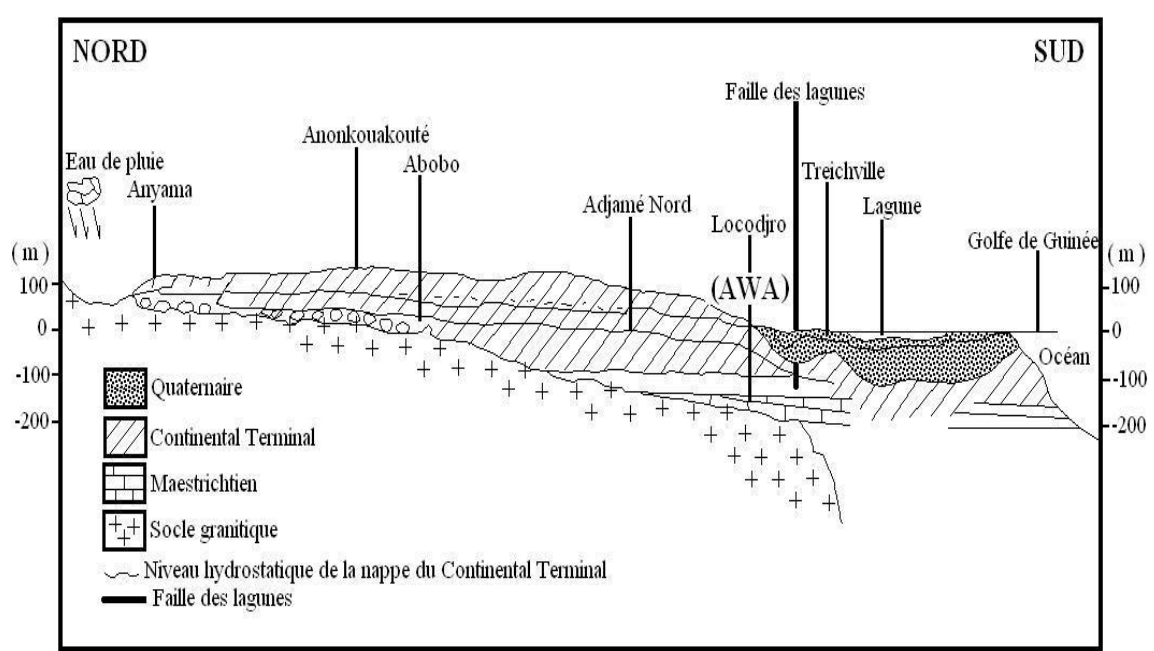

Figure 2 : Coupe géologique NS du bassin sédimentaire côtier (Sadem, 1980, modifié).

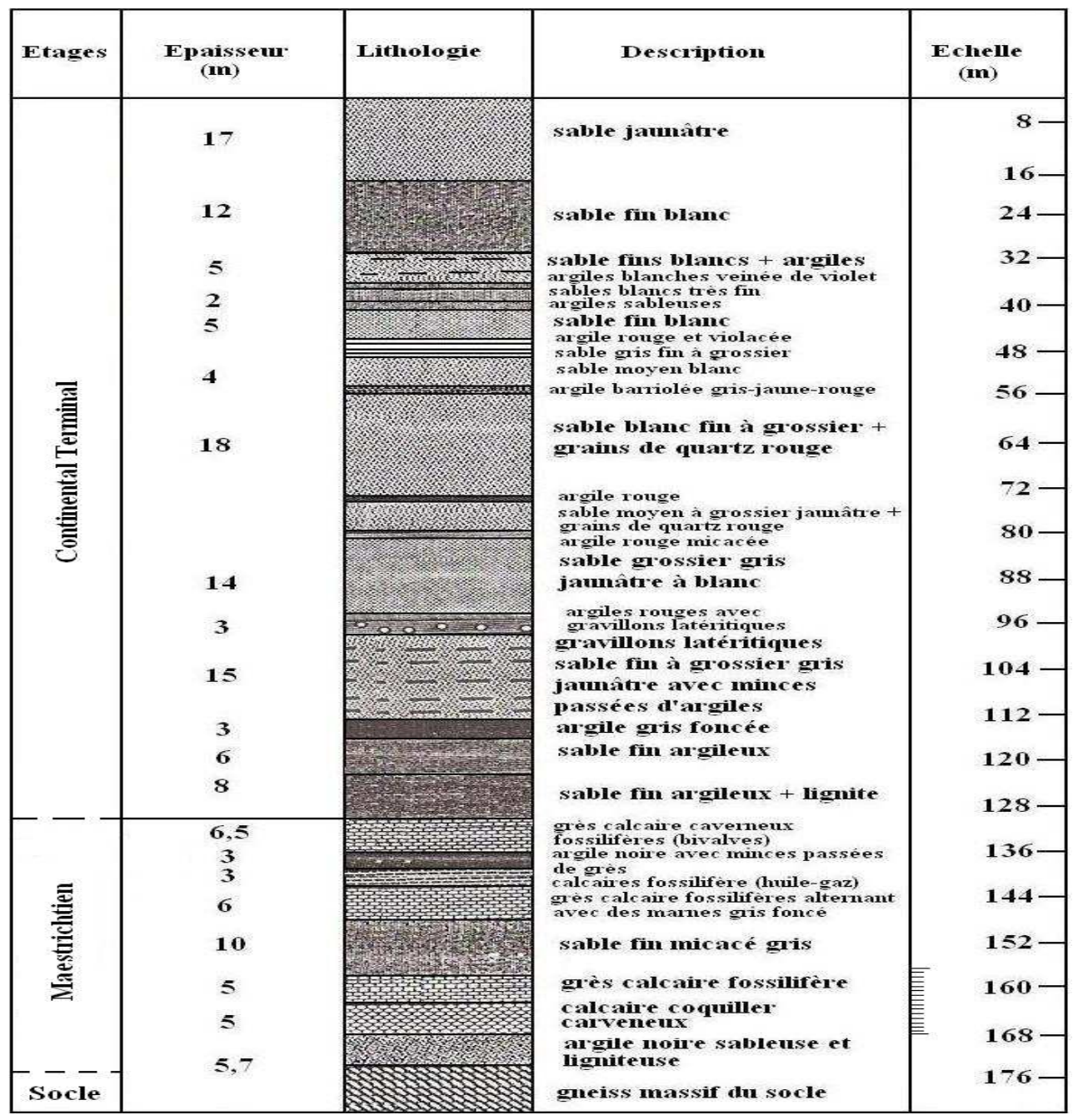

Figure 3 : Log hydrogéologique du forage Awa (Sadem, 1980). 


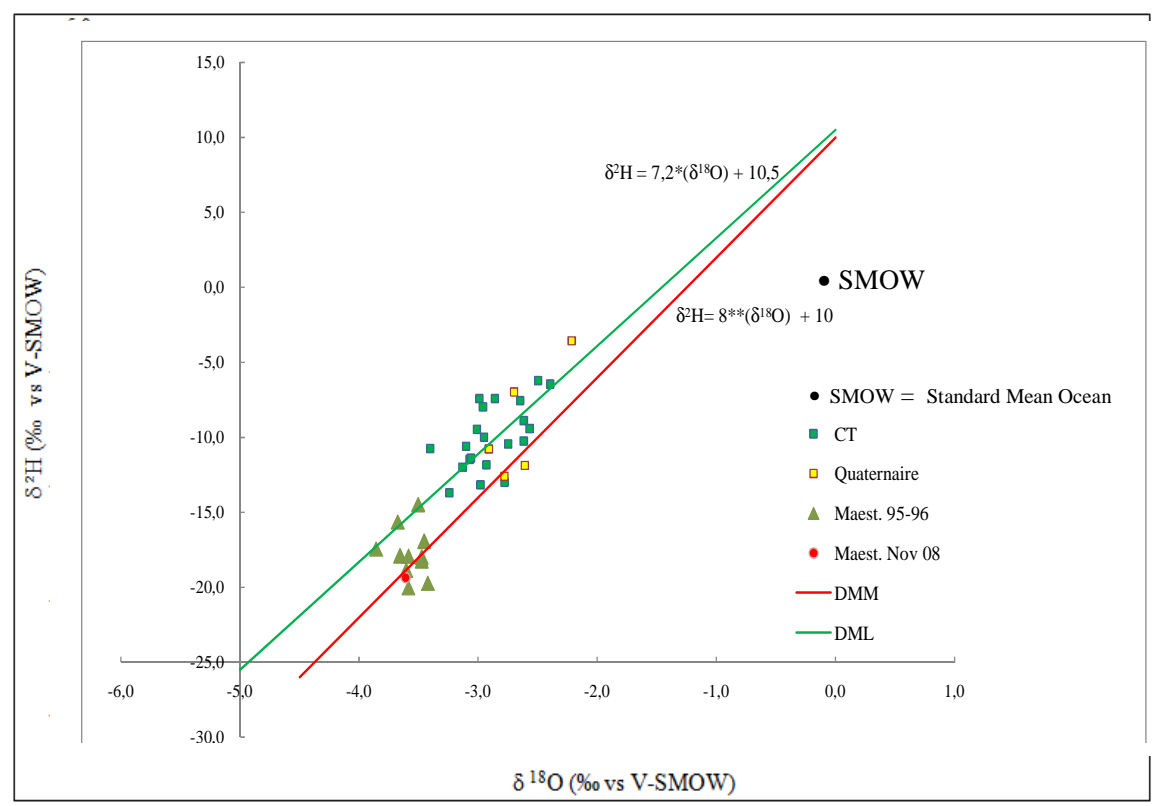

Figure 4: Droite de corrélation $\delta^{2} \mathrm{H} v s \delta^{18} \mathrm{O}$ des eaux des aquifères du bassin sédimentaire côtier de Côte d'Ivoire.

\section{DISCUSSION}

Les eaux du Maestrichtien se distinguent de l'ensemble des eaux souterraines du bassin sédimentaire côtier ivoirien (Quaternaire et Continental Terminal) par un $\mathrm{pH}$ neutre $(\mathrm{pH}=6,86)$, une alcalinité plus forte $\left(205 \mathrm{mg}^{-1} \mathrm{l}^{-1}\right)$, une conductivité électrique plus élevée $\left(394 \mu \mathrm{S} . \mathrm{cm}^{-1}\right)$ et une minéralisation totale élevée de $331 \mathrm{mg} \cdot \mathrm{l}^{-1}$.

De manière générale, les concentrations des anions majeurs mesurées sur les eaux des campagnes de septembre 1995 et d'août 1996 varient très peu ou presque pas contrairement à celles des eaux du CT et du Quaternaire (de la même période) qui indiquent un changement des teneurs en éléments chimiques suite à une arrivée d'eau dans l'aquifère après la grande saison des pluies (avril à juillet) comme l'indique le bilan hydrique (Oga, 1998). Des analyses plus récentes en ions majeurs sur des eaux du Maestrichtien prélévées en avril 2007 (Blé, 2010) indiquent une similitude des valeurs des paramètres physico-chimiques en dehors de la concentration en calcium et magnésium. L'homogénéité temporelle des paramètres chimiques des eaux du Maestrichtien suggère une recharge peu significative ou une absence de recharge de la nappe, ceci en relation avec la grande profondeur à laquelle se situe cet aquifère et la lithologie des formations traversées par les eaux pendant leur infiltration vers la nappe. Des travaux antérieurs (Loroux, 1978; Aghui et Biémi, 1984) indiquent une alimentation indirecte de l'aquifère du Maestrichtien sans toutefois préciser les zones de recharge. Quant à l'homogénéité spatiale des eaux, elle mériterait d'être vérifiée par de nouveaux forages dans l'aquifère du Maestrichtien.

Sur le diagramme de Piper, les eaux du Maestrichtien d'Abidjan sont classées bicarbonatées calciques comme la plupart des eaux souterraines en Afrique de l'Ouest (Biémi, 1992; Adiaffi, 2008; Oga et al., 2009a). La présence des conditions oxydantes permet de négliger à priori les réactions de type redox dans cet aquifère.

Les indices de deséquilibre négatifs obtenus indiquent que les eaux dans l'aquifère $\mathrm{du}$ Maestrichtien altèrent les minéraux primaires au profit de la kaolinite Ce résultat est similaire à ceux obtenus sur les eaux des aquifères du CT et du Quaternaire du bassin côtier de la Côte d'Ivoire (Oga, 1998; Adiaffi, 2008). L'évolution géochimique de ces eaux peut être résumée par les réactions d'altération des minéraux primaires (équation 1 à 5) indiquées ci-dessous : 


$$
\begin{aligned}
& \mathrm{Ca}\left(\mathrm{Al}_{2} \mathrm{Si}_{2}\right) \mathrm{O}_{8}+2 \mathrm{H}^{+}+\mathrm{H}_{2} \mathrm{O}===>\mathrm{Al}_{2} \mathrm{Si}_{2} \mathrm{O}_{5}(\mathrm{OH})_{4}+\mathrm{Ca}^{2+} \\
& \text { Anorthite } \\
& \text { Kaolinite } \\
& \mathrm{Na}\left(\mathrm{AlSi}_{3}\right) \mathrm{O}_{8}+\mathrm{H}^{+}+4,5 \mathrm{H}_{2} \mathrm{O}===>0,5 \mathrm{Al}_{2} \mathrm{Si}_{2} \mathrm{O}_{5}(\mathrm{OH})_{4}+\mathrm{Na}^{+}+2 \mathrm{H}_{4} \mathrm{SiO}_{4} \\
& \text { Albite } \\
& \text { Kaolinite }
\end{aligned}
$$$$
\mathrm{KAlSi}_{3} \mathrm{O}_{8}+\mathrm{H}^{+}+4,5 \mathrm{H}_{2} \mathrm{O}===>0,5 \mathrm{Al}_{2} \mathrm{Si}_{2} \mathrm{O}_{5}(\mathrm{OH})_{4}+\mathrm{K}^{+}+2 \mathrm{H}_{4} \mathrm{SiO}_{4}
$$$$
\text { Microcline }
$$$$
\text { Kaolinite }
$$

$$
\begin{aligned}
& \mathrm{KAl}_{3}\left[\mathrm{Si} 3 \mathrm{O}_{10}\right](\mathrm{OH})+\mathrm{H}^{+}+2,5 \mathrm{H}_{2} \mathrm{O}===> \\
& \text { Muscovite } \\
& 1,5 \mathrm{Al}_{2} \mathrm{Si}_{2} \mathrm{O}_{5}(\mathrm{OH})_{4}+\mathrm{K}^{+} \\
& \text {Kaolinite }
\end{aligned}
$$$$
\mathrm{Na}\left(\mathrm{AlSi}_{3}\right) \mathrm{O}_{8}+\mathrm{H}^{+}+7 \mathrm{H}_{2} \mathrm{O}==>\mathrm{Al}(\mathrm{OH})_{3}+\mathrm{Na}^{+}+3 \mathrm{H}_{4} \mathrm{SiO}_{4}
$$$$
\text { Albite }
$$$$
\text { Gibbsite }
$$

Les bicarbonates dans les eaux souterraines de l'aquifère du Maestrichtien sont essentiellement liés aux équilibres calcocarboniques mettant en jeu la phase gazeuse et la phase liquide. Pour ces eaux, deux cas sont à considérer :

-L'aquifère n'est pas carbonaté : le $\mathrm{CO}_{2}$ $\mathrm{du}$ sol imposerait seul sa signature isotopique à la phase liquide. L'âge calculé par la décroissance radioactive sans correction de l'activité mesurée est de $11000 \pm 125$ ans (âge limite).

-L'aquifère est carbonaté : le carbone en solution a alors deux origines, l'une biogène et l'autre minérale. Le calcul de l'âge de ces eaux souterraines nécessite des modèles de correction puisque le Carbone Inorganique Total Dissout (CITD) a deux origines (Fontes, 1992). Ce qui nécessite une correction de l'activité ${ }^{14} \mathrm{C}$ du CITD, liée à la mise en solution de carbonate par les eaux du forage $\mathrm{du}$ Maestrichtien qui présentent, par ailleurs, un indice proche de la saturation vis-à-vis calcite (I.S. $=-0,39)$ et une teneur en ${ }^{13} \mathrm{C}$ de $16,2 \%$ o. L'utilisation de modèles de correction est nécessaire pour une estimation de l'activité initiale $\mathrm{A}_{0}$. L'âge estimé par les différents modèles de correction varie de 6700 à 9900 ans (Tableau 3).

La valeur $\mathrm{du}{ }^{13} \mathrm{C}$ du CITD (-16,2\%o vs PDB) se rapproche de celles des plantes en $\mathrm{C}_{4}$ dont les teneurs isotopiques sont comprises entre -10 et $-16 \%$ vs PDB (Craig, 1954 ; Moulin, 1990). Les plantes avec un cycle à 4 atomes de carbone (cycle de Hatch et Slack) regroupent la plupart des plantes tropicales, la moitié des graminées (la canne à sucre, le sorgho, le mil, le maïs...) et certaines cypéracées. Le forage Awa est situé en milieu urbain donc non cultivé; par endroits, on rencontre entre les habitations ou sur les lots de terrain non construits quelques pieds de maïs ou de canne à sucre.

Les eaux minérales Awa captées dans les formations du Maestrichtien sont dépourvues de tritium $(<0,8$ UT) et indiquent une faible activité en carbone-14 (26,4 pCM). Ceci suggère que l'aquifère du Maestrichtien est susceptible d'être alimenté difficilement, ou avec un certain retard par les eaux d'infiltration.

$\mathrm{La}$ réalimentation très faible ou inexistante de l'aquifère du Maestrichtien indique, par ailleurs, une circulation lente et difficile à travers des formations de faibles perméabilités et une absence d'apports latéraux.

Les teneurs en isotopes stables des eaux prélevées en novembre sont proches des moyennes obtenues sur la période septembre 1995 -août $1996\left({ }^{18} \mathrm{O}=-3,57 \pm 0,13 \%\right.$ ovs $\mathrm{V}$ SMOW et pour le deutérium à ${ }^{2} \mathrm{H}=-17,74 \pm$ $1,63 \%$ o vs V-SMOW) en tenant compte des incertitudes analytiques sur les mesures. Les eaux du Maestrichtien présentent une teneur en oxygène-18 plus appauvrie par rapport à l'ensemble des eaux souterraines du bassin sédimentaire de la Côte d'Ivoire. Il n'y a pas de tendance mesurée à l'enrichissement ou à l'appauvrissement des teneurs au cours de la période d'échantillonnage des eaux du forage Awa. 
Par contre, des travaux effectués au Ghana sur plusieurs ouvrages captant le Maestrichtien à différentes saisons indiquent de faibles variations des teneurs en ${ }^{18} \mathrm{O}$ et en ${ }^{2} \mathrm{H}$ de ces eaux (Jorgensen et BanoengYakubo, 2001).

Sur une droite de corrélation $\delta{ }^{2} \mathrm{H} v s \delta$ ${ }^{18} \mathrm{O}$ (Figure 4), la majorité des eaux du Maestrichtien, se situent autour de la droite météorique mondiale d'équation $\delta^{2} \mathrm{H}=8 \delta^{18} \mathrm{O}$ $+10 \%$ o tandis que les eaux du CT et du Q se positionnent autour de la droite météorique locale d'équation $\delta^{2} \mathrm{H}=(7,3 \pm 0,2) . \delta{ }^{18} \mathrm{O}+$ $(10,6 \pm 0,2) \%$, située elle au dessus de la droite des eaux météoriques mondiales (Oga et al., 2009b). Ceci indique que la recharge de la nappe du Crétacé s'est faite à partir des précipitations différentes de celles qui ont rechagé le CT et le Quaternaire, et que l'eau de recharge comporte une part d'eau issue de vapeur continentale.

Des études antérieures au Ghana (Jorgensen et Banoeng-Yakubo, 2001) et au Togo (Akouvi et al., 2008) indiquent que les eaux souterraines profondes du Maestrichtien se placent près de la droite des eaux météoriques mondiales dans un diagramme $\delta$ ${ }^{2} \mathrm{H} v s \delta{ }^{18} \mathrm{O}$.

Les eaux du Maestrichtien, plus anciennes (6700 et 9900 ans B.P.) et plus appauvries en isotopes lourds $\left({ }^{2} \mathrm{H},{ }^{18} \mathrm{O}\right)$ que celles du CT et du Quaternaire pourraient s'être rechargées dans des conditions climatiques plus froides que l'actuel. Les travaux de Adiaffi et al. (2009) sur les eaux du socle sous couvert de formations $\mathrm{CT}$ indiquent des temps de résidence similaires (de 8000 et 15000 ans). Ces temps correspondent selon l'auteur au début de la période post glaciaire mise en évidence par des études antérieures au Ghana (Talbot et Johannessen, 1992) et au Libéria (Jahns et al., 1998).

L'activité ${ }^{14} \mathrm{C}$ des eaux prélevées en novembre 2008 indique un rajeunissement par rapport à l'activité ${ }^{14} \mathrm{C}$ sur les eaux d'août 1996. Toutefois, la valeur du ${ }^{13} \mathrm{C}$ du CITD ($14,73 \%$ ov $\mathrm{PDB}$ ) est similaire à celle obtenue sur les eaux de 1996 et est comprise dans l'intervalle des teneurs en ${ }^{13} \mathrm{C}$ des plantes (C4) avec un cycle à 4 atomes de carbone $(-10$ $<\delta^{13} \mathrm{C}<-16 \%$ vs $\left.\mathrm{PDB}\right)$. L'activité ${ }^{14} \mathrm{C}$ des eaux du Maestrichtien prélévées en 2008 se rapproche des activités des eaux du socle sous CT prélévées à Kodiakro $\left(\mathrm{A}^{14} \mathrm{C}=35,3 \pm 0,2\right.$ $\mathrm{pCM})$ et à Attinguié $\left(\mathrm{A}^{14} \mathrm{C}=42,3 \pm 0,2 \mathrm{pCM}\right)$ dans la zone de contact «socle-bassin sédimentaire» (Adiaffi et al., 2009). Par ailleurs, les teneurs en ${ }^{13} \mathrm{C}$ des eaux de socle sous couvert sédimentaire à Kodiakro $\left(\delta^{13} \mathrm{C}=-\right.$ $25,9 \%$ vs $\mathrm{PDB})$ et à Attinguié $\left(\delta^{13} \mathrm{C}{ }^{13} \mathrm{C}=-\right.$ $24,3 \%$ o vs PDB) caractérisent les plantes de type C3 témoins de la présence de forêts denses sur ces zones lors de la recharge.

L'enrichissement des teneurs en carbone-13 des eaux du Maestrichtien par rapport à celles de Kodiakro et Attinguié pourrait être attribué soit à une dilution du carbone biogénique avec le carbone minéral soit à l'effet des pompages.

Le qualificatif "eau minérale" attribué aux eaux du forage Awa est lié à la présence dans ces eaux de minéraux et oligo-éléments $\left(\mathrm{Ca}^{2+}\right.$ et $\left.\mathrm{Mg}^{2+}\right)$. Ces eaux ont été enrichies en ces éléments pendant leur long trajet à travers les différentes strates du sous-sol. Ces eaux en partie fossile indiquent également l'absence de contamination visible par intrusion saline d'eau de mer Contrairement aux eaux du Maestrichtien de la Côte d'Ivoire, celles du bassin de Keta au Ghana et celles du Togo sont marquées par une influence marine significative (Jorgensen et Banoeng-Yakubo, 2001 ; Akouvi et al., 2008).

\section{Conclusion}

Les principaux résultats obtenus dans le cadre de cette étude sont resumés ci-dessus:

1- les eaux de la nappe semi-captive du Maestrichtien sont protégées naturellement de tout risque de pollution $\left(\mathrm{NO}_{3}{ }^{-}=0 \mathrm{mg} \cdot \mathrm{L}^{-1}\right)$ par la lithologie des couches sus-jacentes composées essentiellement de sables, d'argiles sableuses et d'argiles et de la profondeur du forage (191 m) ;

2- Le CITD des eaux souterraines captées dans le Maestrichtien a deux origines, une biogénique et une minérale; ceci en conformité avec les données géochimiques liées à la nature géologique de l'aquifère du Maestrichtien (calcaire gréseux).

3- Les eaux captées dans l'aquifère du Maestrichtien sont dépourvues de ${ }^{3} \mathrm{H}$ et indique une faible activité au ${ }^{14} \mathrm{C}(26,4 \mathrm{pcm})$. 
La réalimentation très faible ou inexistante de l'aquifère du Maestrichtien indique une circulation lente à travers des formations de faibles perméabilités et/ou une absence d'apports latéraux.

4- Le forage de la SADEM contient des eaux anciennes probablement marquées par les changements climatiques sur l'Afrique durant les derniers milliers d'années.

En perspective, il sera nécessaire de poursuivre les études sur l'aquifère du Maestrichtien:

i) Pour définir les paramètres hydrodynamiques

ii) Pour vérifier l'absence d'intrusion saline ou de drainance des aquifères sus-jacents sous l'effet des pompages.

iii) Et pour déterminer les causes du rajeunissement des eaux du Maetrichtien mis en évidence par l'activité ${ }^{14} \mathrm{C}$.

\section{REMERCIEMENTS}

L'étude a bénéficié du soutien matériel du CIAPOL à Abidjan (Côte d'Ivoire) et du laboratoire IDES à Orsay (France) pour les analyses chimiques et isotopiques. Nous disons notre reconnaissance aux responsables de ces laboratoires. Nous tenons également à remercier la société Sadem pour sa collaboration, les collègues et tous les rewievers dont les remarques ont amélioré la qualité de ce manuscrit.

\section{REFERENCES}

Aghui N, Biémi J. 1984. Géologie et hydrogéologie des nappes de la région d'Abidjan et risques de contamination. Annales de l'Université Nationale de Côte d'Ivoire série C, 20: 313-347.

Adiaffi B. 2008. Apport de la géochimie isotopique, de l'hydrochimie et de la télédetectionà la connaissance des aquifères de la zone de contact "soclebassin sédimentaire" du sud est de la Côte d'Ivoire. Thèse de doctorat, Université Paris Sud, Orsay, France, p. 217.

Adiaffi B, Marlin Ch, Oga YMS, Massault M, Noret A, Biémi J. 2009. Paleoclimatic and deforestation effect on the chemical and isotopic composition of the coastal fresh groundwater resources of South-
East Ivory Coast. Journal of Hydrology, 369:130-141.

Ahoussi KE, Soro N, Soro G, Lasm T, Oga MS, Zade SP. 2009. Caractérisation de la qualité physicochimique et bactériologique des eaux de puits de la ville d'Abidjan (Côte d'Ivoire). Africa Geoscience Review, 16(3): 203-215.

Akouvi A, Dray M, Violette S,De Marsily, Zuppi GM. 2008. The sedimentary coastal basin of Togo: example of a multilayered aquifer still influenced by a palaeo-seawater intrusion. Hydrogeology Journal, 16: 419-436.

Biémi J. 1992. Contribution à l'étude géologique, hydrogéologique et par télédétection des bassins versants subsahariens du socle précambrien d'Afrique de l'Ouest: hydrostructurale, hydrodynamique, hydrochimie et isotopie des aquifères discontinus de sillons et aires granitiques de la Haute Marahoué (Côte d'Ivoire). Thèse de doctorat ès Sciences Naturelles, Université Nationale de Côte d'Ivoire, p. 480.

Blé LO. 2010. Eaux minérales et non minérales conditionnées pour la consommation humaine en Côte d'Ivoire: Caractérisation physicochimique, potabilité et risque sanitaires. Cas du District d'Abidjan (zone Sud de la Côte d'Ivoire) Thèse de doctorat, Université de Cocody, Côte d'Ivoire, p. 132.

Bouzid M. 1967. Données hydrogéologiques sur le bassin sédimentaire côtier du Dahomey, FAO, (projet DAH3).

Coleman ML, Sherpherd TJ, Durham JJ, Rousse JE, Moore GR. 1982. Reduction of water with zinc for hydrogen isotope analysis. Analytical Chemistry, 54: 993995.

Coly A. 1996. Le systeme fluvio-lacustre du lac de Guiers: étude hydrologique et gestion quantitative intégré. Thèse doctorat $3^{\text {eme }}$ cycle UCAD, Dakar, . p. 350 .

Craig H. 1954. Carbon-13 in plants and relationships between carbon-13 and variations in nature. The Journal of Geology, 62(2):115-141.

DGEA. 2009. Etude de faisabilité des forages manuels-Identification des zones 
potentiellement favorables. http://www. unicef.org/wash/files/Cote_Ivoire_Rappo rt_final_des_zones_favorables_(FINAL). pdf Pratica fondation / Entreprise Works/Unicef avec la collaboration de l'UFR-STRM, Université d'Abidjan. p. 73.

Eichinger E. 1983. A contribution to the interpretation of ${ }^{14} \mathrm{C}$ groundwater ages considering the example of partially confined sandstone aquifer. Radiocarbon, 25: 347-356.

Epstein S, Mayeda TK. 1953. Variations of the ${ }^{18} \mathrm{O} /{ }^{16} \mathrm{O}$ ratio in naturel waters. Geochimica Cosmochimica Acta, 4: 213224.

Evans GV, Otlet R L, Downing A, Monkhouse RA, Rae G. 1979. Some problems in the interpretation of isotop measurements in United-Kingdom aquifers Isotope Hydrology. Proceeding of a Symposium, Neuherberg, IAEASM228/34, 19-23 june 1978. 639-708.

Fontes JCH. 1992. Chemical and isotopic constraints on ${ }^{14} \mathrm{C}$ dating of groundwater. In Radiocarbon. After Four Decades. An Interdisciplinary Perspective. Taylor RE, Long A, Kra RS (eds). Springer-Verlag; 242-261.

Fontes JCH, Garnier JM. 1979. Determination of the initial ${ }^{14} \mathrm{C}$ activity of the Total Dissolved Carbon: a review of the existing models and a new approach. Water Ressources Research, 14(2): 399413.

Fritz B. 1976. Etude thermmodynamique et simulation des réactions entre minéraux et solutions. Applications à la géochimie des altérations et des eaux continentales. Sciences géologiques mémoire $\mathrm{n}^{\circ}$. 41, 1975, Université Louis Pasteur de Strasbourg, p.152.

Guerin-Villeaubreuil G. 1962. Hydrologie en Côte d'Ivoire. Mémoires du Bureau de Recherches Géologiques et Minières; Editions techniques, 20, p. 43.

Ingerson E, Pearson JF. 1964. Estimation of âge and rate of motion of groundwater by ${ }^{14} \mathrm{C}$ method. In Atmosphere and Nuclear Geochemmistry (Eds. Maruzen). Recent Researches in the fields of
Hydrolosphere, Sugarawa Festival, Tokyo; 263-283.

Jahns S, Hüls M, Sarnthein M. 1998. Vegetation and climate history of west equatorial Africa based on a marine pollen record off Liberia (site GIK) covering the last 400,000 years. Review of Paleobotany and Palynology, 102: 277288.

Jorgensen NO, Banoeng-Yakubo BK. 2001. Environmental isotopes $\left({ }^{18} \mathrm{O},{ }^{2} \mathrm{H}\right.$, and ${ }^{87} \mathrm{Sr} /{ }^{86} \mathrm{Sr}$ ) as a tool in groundwater investigations in the Keta Basin, Ghana. Hydrogeology Journal, 9: 190-201.

Jourda JP, Kouamé KJ, Saley MB, Kouadio BH, Oga YS, Deh S. 2006. Contamination of the Abidjan Aquifer by sewage : an assessment of extent and strategies for protection. In "Groundwater Pollution in Africa", Yongxin Xu, Brent Ushers (eds). Taylor \& Francis Group: Balkema,Great-Britain; 291-300.

Kouadio LP, Abdoulay BS, Jourda P, Loba MA, Rambaud M. 1998. Conséquences de la pollution urbaine sur la distribution d'eau d'alimentation publique à Abidjan. Cahiers de l'Association Scientifique Europenne pour l'Eau et la Santé, 3(1).

Leneuf N, Berthelot D. 1966. Données nouvelles sur le contact socle-Continental Terminal. Comptes Rendus de la Société Geologique de France. Fascicule, 8: 283284.

Loroux BFE. 1978. Contribution à l'étude hydrogéologique du bassin sédimentaire côtier de Côte d'Ivoire. Thèse Université de Bordeaux I, France, p. 93.

Martin L. 1973. Carte sédimentologique $d u$ Plateau Continental de Côte d'Ivoire; Note explicative no 48. ORSTOM, CRO: Abidjan ; 25.

Mook WG. 1976. The dissolution-exchange model for dating groundwater with ${ }^{14} \mathrm{C}$. In Interpretation of Environmental Isotope and Hydrochemical data in Groundwater Hydrology. IAEA: Vienna; 213-225.

Mott Mc. Donald International. BCEOM. SOGREAH. ORSTOM. 1993. Evaluation hydrologique de l'Afrique subsahérienne. Pays de l'Afrique de l'ouest: 
Rapport de pays : Togo (BM/PNUD/BAD/MFC) 01/1993.

Moulin M. 1990. Genèse des minéraux secondaires en zone non saturée carbonatée : étude géochimique et isotopique de la zone non saturée de la craie de Champagne. Thèse de doctorat, Université de Paris XI, France, p. 194.

Oga YMS. 1998. Ressources en eaux souterraines dans la région du Grand Abidjan (Côte d'Ivoire): Approches hydrochimique et isotopique. Thèse de doctorat, Université Paris Sud, Orsay, France, p. 240.

Oga YMS, Sacchi E, Zuppi GM. 2007. Origin and effects of nitrogen pollution in groundwater traced by $\delta^{15} \mathrm{~N}_{-} \mathrm{NO}_{3}$ and $\delta^{18} \mathrm{O}-\mathrm{NO}_{3}$ : the case of Abidjan (Ivory Coast). Advances in isotope hydrology and its role in sustainable water resources management (HIS-2007) Proceedings of a symposium Vienna 21 - 25 mai 2007, 1: 139-147.

Oga MS, Marlin Ch, Njitchoua R, Dever L et Filly A. 2008. Hydrochemical and isotopic characteristics of Coastal groundwater near Abidjan (Southern Ivory Coast). In Applied Groundwater Studies in Africa. IAH Selected Papers on Hydrogeology (vol. 13), Adelana SMA, MacDonald AM (eds). CRCPress/ Balkema: Leiden, The Netherlands; 371389.

Oga MS, Lasm T, Yao KT, Soro N, Saley MB, Dongo K, Gnamba FM. 2009a. Caractérisation chimique des eaux des aquiferes de fracture : cas de la région de Tiassalé en Côte d'Ivoire. European Journal of Scientific Research, 31(1) : 7287.

Oga YMS, Njitchoua R, Marlin Ch, Dever L, Filly A. 2009b. Variations of heavy isotopic contents $\left(\delta^{18} \mathrm{O}\right.$ and $\left.\delta^{2} \mathrm{H}\right)$ in individual rainfall events in Abidjan, Ivory Coast. Journal des Sciences et Technologies, 8(1): 25-34.

Rollins L. 1987. PC WATEQ DAGEN : an IBM and PC-compatibles adaptation of WATEQF. User's guide, Woodland, California.
SADEM. 1980. Eau du forage de Locodjro. Société des eaux minérales de Côte d'Ivoire.

Salem O, Vissier JH, Dray M, Gonfiantini R. 1980. Groundwater flow patterns in the western Lybian Arab Jamahiriaya. In Arid-Zone Hydrology : Investigations with Isotope Techniques. IAEA: Vienna; 165-179.

Slansky. 1962. Contribution à l'étude géologique du basin sédimentaire côtier (BSC) du Dahomey et du Togo. Mémoire Bureau Recherche Minière, France, p. 270.

SOGREAH Ingénierie. 1996. Etude de la gestion et de la protection de la nappe assurant l'alimentation en eau potable d'Abidjan; Etude sur modèle mathématique ; Rapports de phase 1 et 2 , République de Côte d'Ivoire, Ministère des Infrastructures Economiques, Direction et Contrôle des Grands Travaux (DCGTx, actuel BNETD).

Sow E. 1992. Etude sédimentologique et revision chronostratigraphique du Crétacé Terminal du Horst de N'Diass (sénégal occidental), Thèse de doctoract $3^{\text {ème }}$ cycle, UCAD, Dakar, p. 152

Stumm W, Morgan JJ. 1981. Aquatic Chemistry : an Introduction Emphasizing Chemical Equilibria in Waters. J. Wiley \& Sons: New-York; 780.

Tamers MA. 1967. Radiocarbon ages of groundwater in an arid zone confined aquifer; Isotope Techniques in the Hydrological Cycle, 11. American Geophysical Union Monograph; 143-152.

Talbot MR, Johannessen T. 1992. A high resolution paleoclimatic record for the last 27,500 years in tropical West Africa from the carbon and nitrogen isotopic composition of lacustrine organic matter. Earth and Planetary Sciences Letters, 110: 23-37. 